\title{
Kommt der Aldiminform der glykosylierten Hämoglobine beim Diabetes mellitus eine diagnostische und/oder prognostische Wertigkeit zu?
}

\author{
G. Sachse, A. Schornstein, H. Laube, K. Foderlin \\ III. Medizinische Universitäts-Klinik und Poliklinik, Gießen
}

\section{Zusammenfassung:}

Die vorliegende Studie untersucht die Bedeutung der labilen Aldiminform bei der Bestimmung glykosilierter Hämoglobine. Bei 50 Typ I (insulinbedürftigen) Diabetikern wurden engmaschige Blutzuckertagesprofile (nüchtern 8.30, 11.00, 12.30, 15.00, 17.00, 19.00, 20.00, 22.00 Uhr) erstellt. Am ersten und letzten Tag des stationären Aufenthaltes sowie 3 Wochen nach der Entlassung erfolgte die Bestimmung der HbA $\mathrm{A}_{\mathrm{a}-\mathrm{c}}$-Werte. Bei 10 Patienten mit extrem labiler Stoffwechsellage bestimmten wir parallel zu den engmaschigen Blutzuckertagesprofilen sogenannte

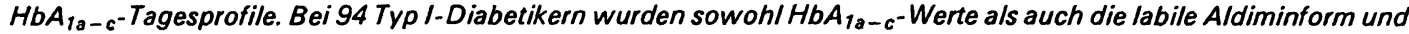
die Ketoaminform getrennt bestimmt. Wir sahen keine Korrelation zwischen mittlerer Schwankungsbreite im Blutzuckertagesprofil (MAGE) respektive $H_{b A_{1 a-c}}$ Werten, z.T. fast im Normbereich liegende $H_{b A_{1 a-c}-W e r t e}$

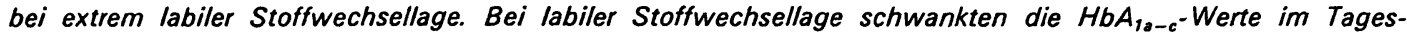
verlauf signifikant entsprechend den aktuellen Blutzuckerwerten. Die Aldiminform korrelierte signifikant zur MAGE- sowie zu bereits bestehender diabetischer Neuropathie bzw. Retinopathie.

\section{Schlüsse/wörter:}

Diabetes mellitus - glykosilierte Hämoglobine - Aldiminform - Labilität der Stoffwechsellage - diabetische Spätkomplikationen

\section{Summary:}

To evaluate the importance of the labile aldimine fraction the following studies were done.

In 50 type-1-insulindependent diabetics we determined serial blood glucose profiles (fasted, 8.30 a.m., 11.00 a.m., 12.30 a.m., 3.00 p.m., 5.00 p.m., 7.00 p.m., 8.00 p.m., 10.00 p.m.). HbA ${ }_{1 a-c}$ was determined on the first and the last day of the stationary stay and again 3 weeks after demission from the hospital. Furthermore we studied 10 type-1diabetics with extremely labile carbohydrate metabolism. Again serial blood glucose profiles and in addition serial $H b A_{1 a-c}$-profiles were determined.

In a third study we determined $H b A_{1 a-c}$ and the stabile ketoamin and the labile adlimine in 94 type 1-diabetics. There was no correlation between MAGE respectively $\mathrm{HbA}_{1 a-c}$ in labile diabetics. $\mathrm{HbA}_{1 a-c}$-values changed significantly allover the day corresponding to actual blood glucose values. The labile aldimine fraction showed a significant correlation to the MAGE and furthermore to diabetic neuropathy and retinopathy.

Keywords:

Diabetes mellitus - glycosilated hemoglobins - aldiminfraction - lability of carbohydrate metabolism - diabetic late complications

\section{Einleitung}

Die Bestimmung der glykosilierten Hämoglobine $\left(\mathrm{HbA}_{1 \mathrm{a}-\mathrm{c}}\right)$ soll Auskunft über die Güte der diabetischen Stoffwechselführung geben. $\mathrm{HbA}_{1 \mathrm{a}-\mathrm{c}}$ hat wie normales Hämoglobin ein Molekulargewicht von 64000. Es besitzt die gleiche Aminosäuresequenz und liegt auch in der Tetramerform (Alpha-2 Beta-2) vor. Nach der Reduktion von $\mathrm{HbA}_{1 \mathrm{a}-\mathrm{c}}$ mit Natriumborhydrid $\left(\mathrm{NaBH}_{4}\right)$ erhält man eine Schiffsche Base, die an das n-terminale Ende der Beta-Kette gebunden ist (1). Dieses relativ instabile Aldimin lagert sich nach Amadori zum stabilen. Ketoamin um (2). Bei der stabilen Verbindung am n-termina- len Ende der Beta-Kette handelt es sich um eine 1-Amino-1desoxyfructose $(3,4)$. In zahlreichen Studien konnte gezeigt werden, daß die stabile Ketoaminform der glykosilierten Hämoglobine die mittlere Blutzuckerhöhe der zurückliegenden 4-6 Wochen integriert (5-13). Im Jahre 1977 wurde erstmals darauf hingewiesen, daß in vivo bereits eine nur kurzzeitige Erhöhung der Blutglucosekonzentration einen Anstieg der glykosilierten Hämoglobine bewirke (14). Fernerhin konnte gezeigt werden, daß die Inkubation von Vollblut oder Erythrozytenhämolysat mit Glucose schon nach ca. 2 Std. zu einem Anstieg der $\mathrm{HbA}_{1 \mathrm{a}-\mathrm{c}}-$ Konzentration führte $(15,16)$.' Es ergab sich hieraus die Konsequenz, daß bei der 


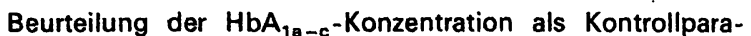
meter der diabetischen Stoffwechsellage diese schnellen Schwankungen der instabilen Fraktion (Aldiminform) berücksichtigt werden müßten (17).

Die vorliegende klinische Studie stellt die Bedeutung dieser labilen Aldiminform zur Diskussion: ist der Aldiminanteil in der klinischen Routinediagnostik zu vernachlässigen, sollte er als „Störgröße" meßtechnisch eliminiert werden, oder kommt inm eine diagnostische und/oder prognostische Wertigkeit zu?

\section{Methodik}

Es wurden insgesamt 3 unterschiedliche Patientenkollektive untersucht.

\section{Studie I}

Zunächst untersuchten wir 50 Typ-I-Diabetiker während eines stationären Krankenhausaufenthaltes. Das Alter der Patienten betrug 17-59 (37 \pm 21$)$ Jahre, die Diabetesdauer $1-22(11 \pm 8)$ Jahre. Wir bestimmten täglich über 2 Wochen sog. engmaschige Blutzuckertagesprofile (nüchtern, 8.30, $11.00,12.30,15.00,17.00,19.00,20.00,22.00 \mathrm{Uhr}$ ) und errechneten aus den Einzelwerten sowohl die mittleren Blutzuckertageswerte (MBG) als auch die mittlere Schwankungsbreite im Blutzuckertagesprofil (MAGE). $\mathrm{HbA}_{1 \mathrm{a}-\mathrm{c}}$ (Fa. BIORAD) wurde mittels Mikrosäulenchromatographie bei sämtlichen Patienten am Aufnahmetag, zur Entlassung und nochmals 3 Wochen nach der Entlassung bestimmt (Zusammenfassung der Daten in Tab. 1).

\section{Studie /I}

In dieser Studie untersuchten wir 10 Typ-I-Diabetiker mit extrem labiler Stoffwechsellage. Das Alter dieses Kollektivs betrug 16-31 (26 \pm 7$)$ Jahre, die Diabetesdauer 4-11 $(7 \pm 3)$ Jahre. Als Kontrollgruppe dienten Typ-2-Diabetiker mit stabiler Stoffwechsellage. Bei diesen Patienten bestimmten wir wiederum engmaschige Blutzuckertagesprofile und parallel dazu sog. $\mathrm{HbA}_{1 \mathrm{a}-\mathrm{c}}$-Tagesprofile (Zusammenfassung der Daten in Tab. 2).

\section{Studie III}

Sie wurde unter ambulanten Bedingungen durchgeführt. Bei 94 Typ-I-Diabetikern im Alter von 21 bis $72(43 \pm 18)$ Jahren, Diabetesdauer 3-26 (16 \pm 10$)$ Jahre bestimmten wir jeweils ein engmaschiges Blutzuckertagesprofil mit Ermittlung der mittleren Schwankungsbreite im Tagesverlauf (MAGE). Am gleichen Tag erfolgte die Bestimmung der glykosilierten Hämoglobine mit Elimination und Bestimmung der labilen Aldiminform (Aldimineliminator Fa. Panchem). Von diesen Patienten wiesen 37 eine diabetische Retinopathie, 29 eine diabetische Neuropathie (peripher und/oder autonom) auf (Zusammenfassung der Daten in Tab.3).

\section{Statistische Auswertung}

Für die Beurteilung von Mittelwertdifferenzen wurde unter Voraussetzung einer Normalverteilung der Student's t-Test für unverbundene Stichproben angewandt. Als signifikant wurde die Differenz zweier Werte angesehen, wenn bei zweiseitiger Prüfung $\mathrm{p}<0,01$ war (18).
Tab. 1: Zusammenfassung der Patientendaten aus Studie 1

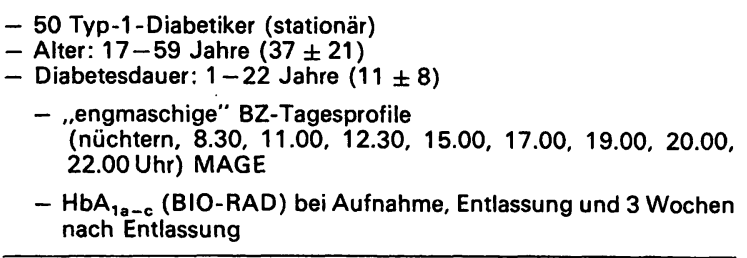

Tab. 2: Zusammenfassung der Patientendaten aus Studie 2

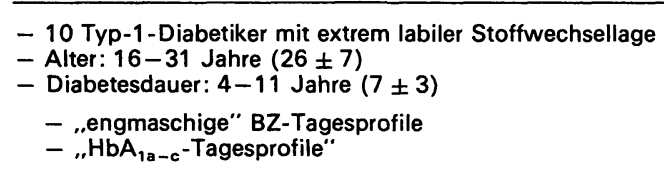

Kontrollgruppe: 10 Typ-2-Diabetiker mit stabiler Stoffwechsellage

Tab. 3: Zusammenfassung der Patientendaten aus Studie 3

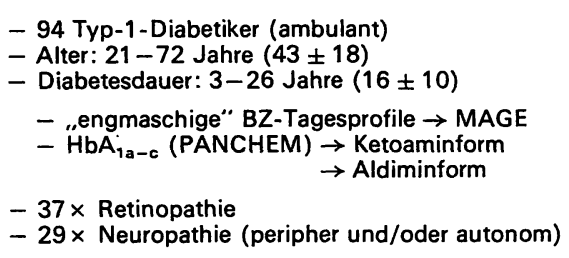

\section{Ergebnisse}

Abb. 1 zeigt den Zusammenhang zwischen $\mathrm{HbA}_{1 \mathrm{a}-\mathrm{c}}$ und mittleren Blutzuckertageswerten. Wir sehen erwartungsgemäß eine gute Korrelation $(r=0,674 ; p<0,005)$. Setzt man jedoch die gemessenen $\mathrm{HbA}_{1 \mathrm{a}-\mathrm{c}}$-Werte in Beziehung zur MAGE als Maß für die Labilität der diabetischen Stoffwechsellage, so zeigt sich hier eine fehlende Korrelation (Abb. 2).

In den Tab. 4 und 5 sind die Blutzuckerwerte bzw. $\mathrm{HbA}_{1 \mathrm{a}-\mathrm{c}^{-}}$ Profile der 10 extrem labilen Typ-I-Diabetiker dargestellt. Es zeigen sich extrem starke Blutzuckerschwankungen im Tagesverlauf, denen Schwankungen der $\mathrm{HbA}_{1 \mathrm{a}-\mathrm{c}}$-Tageswerte bis zu $2 \%$ zuzuordnen sind.

Abb. 3 gibt im Vergleich hierzu die $\mathrm{HbA}_{1 \mathrm{a}-\mathrm{c}}$ - und Blutzuckermittelwerte des Kontrollkollektivs (10 stabile Typ-2Diabetiker) wieder, bei denen keine signifikanten Schwankungen im Tagesverlauf auftreten.

Abb. 4 zeigt den Zusammenhang zwischen labilem Aldiminanteil und MAGE bei 94 Typ-1-Diabetikern. Hier findet sich interessanterweise eine signifikante Korrelation $(r=0,683$; $p<0,005)$ worauf in der Diskussion noch näher eingegangen wird. Abb. 5 ist der Zusammenhang zwischen bereits bestehenden diabetischen Spätkomplikationen (Retinopathie, Neuropathie) und dem Anteil der labilen Aldiminform wiedergegeben. Es zeigt sich, daß Patienten mit bestehenden Spätkomplikationen signifikant höhere Aldiminwerte aufweisen, als solche ohne Spätkomplikationen ( $p<0,005$ resp. $p<0,001$ ). 


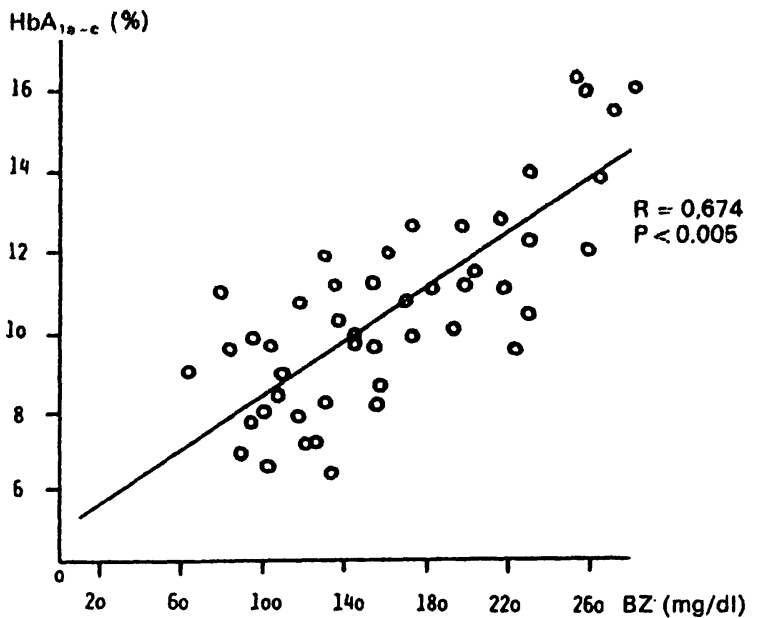

Abb. 1: Korrelation von $\mathrm{HbA}_{1 \mathrm{a}-\mathrm{c}}$ mit den mittleren Blutzukkertageswerten

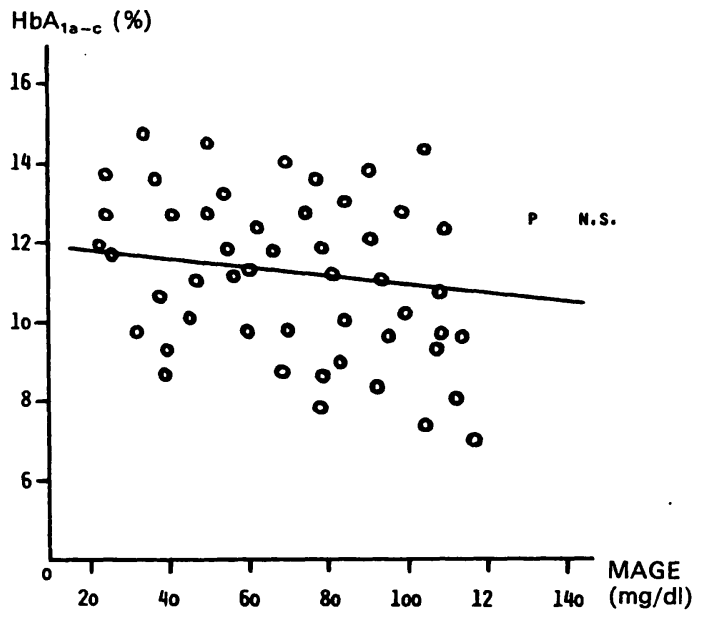

Abb. 2: Fehlende Korrelation der $\mathrm{HbA}_{1 a-c}$-Werte zur MAGE

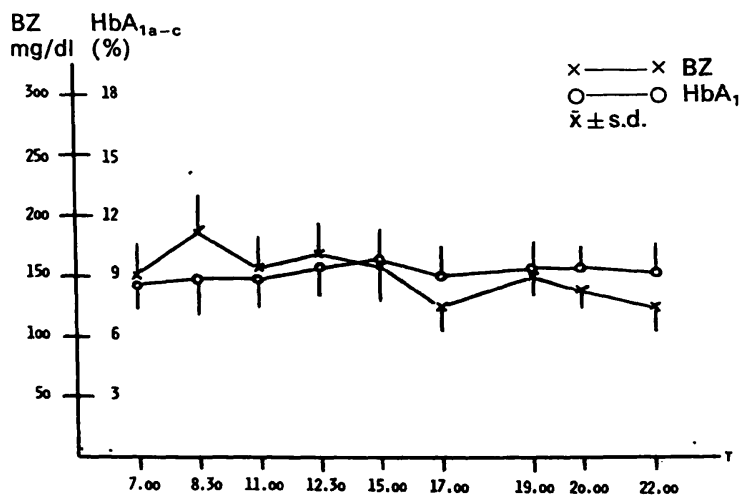

Abb. 3: Blutzucker- und $\mathrm{HbA}_{1 a-c}$-Werte von stabilen Typ-2Diabetikern
Aldimin (\%)

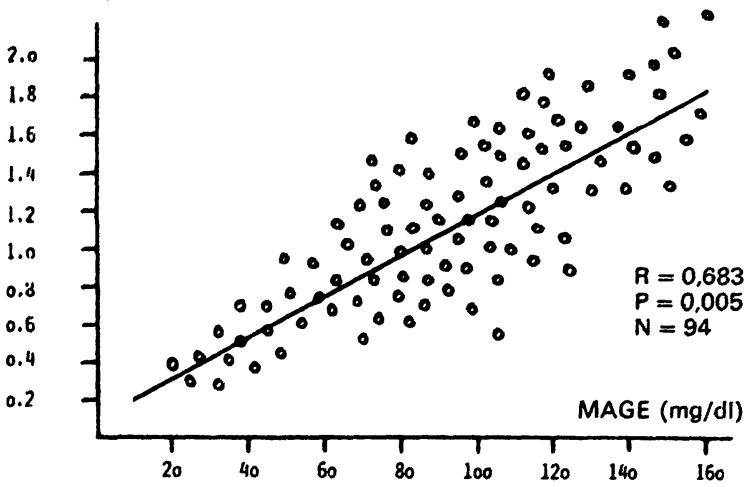

Abb. 4: Korrelation des labilen Aldiminanteils zur MAGE

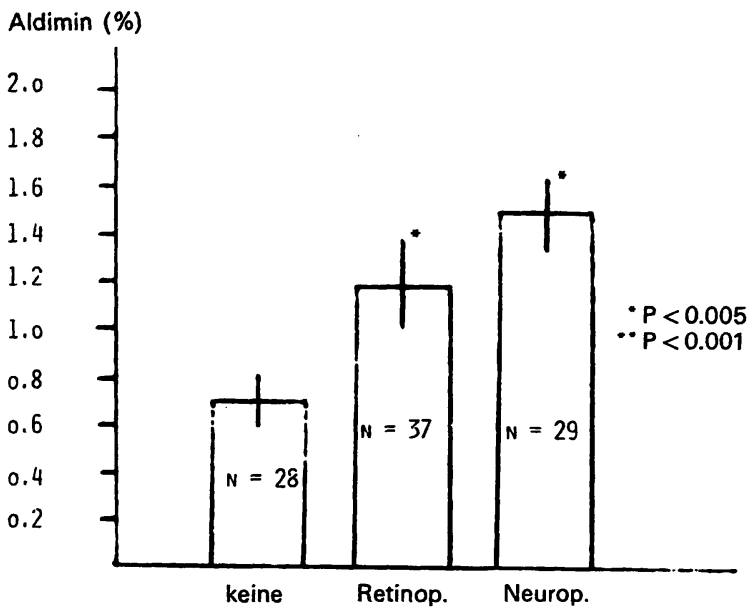

Abb. 5: Zusammenhang zwischen bereits bestehenden diabetischen Spätkomplikationen und dem Anteil der labilen Aldiminform?

\section{Diskussion}

Unsere Untersuchungen zeigen, daß die Bestimmung der Gesamtfraktion der glykosilierten Hämoglobine zwar Aufschluß über die mittlere Blutzuckerhöhe gibt, nicht aber zur MAGE als Maß für die Schwankungsbreite und damit die Labilität der diabetischen Stoffwechsellage korreliert. Die fehlende Korrelation läßt sich dadurch erklären, daß mit der MAGE eine zweidimensionale Meßgröße, nämlich die Schwankungen der Blutzuckerwerte nach oben und unten erfaßt wird, wohingegen ein Anstieg der glykosilierten Hämoglobine.ja nur im Rahmen ansteigender Blutzuckerwerte erfolgt. Es erscheint somit zunächst nicht sinnvoll diese beiden Meßgrößen zueinander in Korrelation zu setzen. Andererseits ist zu überlegen, ob die glykosilierten Hämoglobine wirklich uneingeschränkt als Langzeitparameter der diabetischen Stoffwechsellage bezeichnet werden sollten, wenn sie zwar die mittlere Blutzuckerhöhe integrieren, aber kein $\mathrm{Ma}$ für die Labilität der diabetischen Stoffwechsellage darstellen. Diese Einśchränkung der Aussagefähigkeit der glykosilierten Hämoglobine erscheint wichtig, da bis jetzt nicht sicher gesagt werden kann, inwieweit zum einen insgesamt zu hohe mittlere Blutzuckenwerte, zum anderen aber häufige Blut- 
Tab. 4: Blutzuckerwerte der 10 Patienten mit extrem labiler Stoffwechsellage

\begin{tabular}{|c|c|c|c|c|c|c|c|c|c|}
\hline Patient & nüchtern & 8.30 & 11.00 & 12.30 & 15.00 & 17.00 & 19.00 & 20.00 & $22.00 \mathrm{~h}$ \\
\hline $\begin{array}{r}1 \\
2 \\
3 \\
4 \\
5 \\
6 \\
7 \\
8 \\
9 \\
10\end{array}$ & $\begin{array}{r}103 \\
227 \\
212 \\
299 \\
123 \\
164 \\
143 \\
92 \\
197 \\
62\end{array}$ & $\begin{array}{l}331 \\
374 \\
378 \\
443 \\
328 \\
355 \\
322 \\
299 \\
398 \\
259\end{array}$ & $\begin{array}{l}212 \\
116 \\
286 \\
307 \\
300 \\
296 \\
200 \\
138 \\
256 \\
148\end{array}$ & $\begin{array}{l}138 \\
198 \\
159 \\
138 \\
246 \\
405 \\
249 \\
215 \\
205 \\
307\end{array}$ & $\begin{array}{r}197 \\
335 \\
68 \\
196 \\
181 \\
334 \\
78 \\
307 \\
319 \\
336\end{array}$ & $\begin{array}{l}315 \\
403 \\
217 \\
388 \\
317 \\
308 \\
204 \\
368 \\
221 \\
369\end{array}$ & $\begin{array}{l}358 \\
291 \\
305 \\
112 \\
312 \\
164 \\
337 \\
201 \\
117 \\
384\end{array}$ & $\begin{array}{l}298 \\
207 \\
336 \\
317 \\
289 \\
200 \\
344 \\
126 \\
204 \\
197\end{array}$ & $\begin{array}{r}146 \\
255 \\
200 \\
207 \\
197 \\
72 \\
156 \\
300 \\
107 \\
98\end{array}$ \\
\hline
\end{tabular}

Tab. 5: $\mathrm{HbA}_{1 a-c}$-Profile der 10 Patienten mit extrem labiler Stoffwechsellage

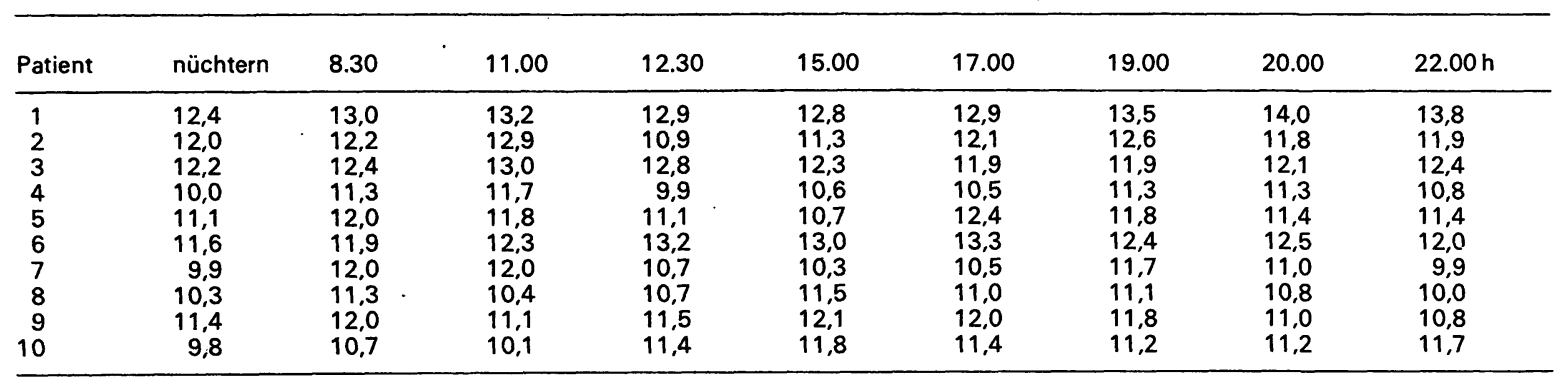

zuckerschwankungen zur Entstehung diabetischer Spätkomplikationen beitragen können. Unseres Erachtens ist die Definition einer langfristig guten diabetischen Stoffwechsellage nur über die Bestimmung der Gesamtfraktion glykosilierter Hämoglobine nicht ausreichend. Frühere Untersuchungen ließen vermuten, daß die Ketoaminkonzentration der Konzentration an instabilen Aldiminen proportional sei (17). Dem widersprechen die Ergebnisse dieser Studie, daß nicht die Gesamtfraktion der glykosilierten Hämoglobine wohl aber der Aldiminanteil zur MAGE korreliert. Diese zunächst widersprüchlich erscheinenden Ergebnisse wären dann zu erklären, wenn wie bereits von anderen Autoren vermutet $(13,19,20)$ auch eine raschere Dissoziation der stabilen Ketoaminform erfolgen würde. Welche Faktoren eine solche Dissoziation fördern oder die Umlagerung des instabilen Aldimins in die stabile Form verhindern, ist bis jetzt noch nicht geklärt. Möglicherweise kommt hier dem pH-Wert des Reaktionsmilieus eine ähnliche Bedeutung zu wie bei der Bildung des instabilen Aldimins selbst.

Die Tatsache, daß Diabetiker mit bereits bestehenden diabetischen Spätkomplikationen signifikant höhere Aldiminanteile aufwiesen als Patienten ohne Spätkomplikationen und, wie gezeigt, der Aldiminanteil zur Labilität der diabetischen Stoffwechsellage korrelierte, wirft erneut die Frage auf, ob und inwieweit Schwankungen der diabetischen Stoffwechsellage das Entstehen diabetischer Spätkomplikationen begünstigen können. Ob in diesem Fall der Aldiminform eine prognostische Wertigkeit bezüglich des Auftretens diabetischer Spätkomplikationen zukäme, müßten weitere Untersuchungen zeigen. Eine Vernachlässigung oder Elimination des Aldiminanteils als .Störgröße" erscheint uns aufgrund der vorgelegten Ergebnisse verfrüht. Die Bedeutung der Aldiminform sollte einer weiteren kritischen Prüfung unterzogen werden.

Schrifnum

1. HOLMQUIST, W. R., SCHROEDER, W. A.: A new terminal blocking group involving a Schitf base in hemoglobin $A_{1 c}$. Biochemistry 5. 2489-2503 (1966).

2. HODGE JE. The Amodori rearranoement Advancos in Carbohydrate. Chem. 10, 169205 (1955).
3. BUNN, H. F., HANEY, D. N., GABBAX, K. H., GALLOP, P. M.: Further identification of the nature and linkage of the carbohydrate in hemoglobin $A_{10}$. Biochem. Biophys. Res. Com. 67 No. 1, 103-109 (1975).

4. KÓENIG, R.J., BLOBSTEIN, S. H., CERAMI, A.: Structure of carbohydrate of hemoglobin $A_{1 c}$. J. Biol. Chem. 252, 2992-2997 (1977).

5. HUISMAN, T. H. J., DOZY.A. M.: Studies on the heterogeneity of hemoglobin V. Binding of hemoglobin with oxidized glutathione. J. Lab. Clin. Med. 60 No. 2, 302-319 (1962). 6. RAHBAR, S.: An abnormal hemoglobin in red cells of diabetics. Clin. Chem. Acta 22, 296-298 (1968).

7. TATTERSALL, R. B., PYKE, D. A., RANNEY, H. M., BRUCKHEIMER, S. M.: Hemoglobin components in diabetes mellitus: studies in identical twins. N. Engl. J. Med. 293, 11711173 (1975).

8. KOENIG, R. J., ARAUJO, D. C., CERAMI, A.: Increased hemoglobin $A_{1 c}$ in diabetic mice. Diabetes 25, 1-5.(1976a).

G KOENIG, R. J., PETERSON, C. M. KILO, C., CERAMI, A. WILLIAMSON, J. R. Hemoglobin $A_{1 c}$ as an indicator of the degree of glucose intolerance in diabetes. Diabetes 25

10. KOENIG, R. J., PETERSON, C. M., JONES, R. L., SAUDEX, C., LEHRMAN, M. CERAMI, A. Correlation of glucose regulation and hemoglobin $A_{1 c}$ in diabetes mellitus. . 295 417-420 (1976C).

11. CERAMI, A., KOENIG, R.J., PETERSON, C. M.: Hemoglobin $A_{1 c}$ and diabetes mellitus. B. Jaematol. 38, 1-3 (1978)

12. GONEN B. RUBENSTEIN, A H ROCHMAN, H. TANEGA S. P. HORWITZ D L. Hemoglobin $A_{1}$ : an indicator of the metabolic control of diabetic patients. Lancet $2,734-$ (1977).

13. GABBAY K. H. HASTY K BRESLOW, J.L. ELLISON, R. C., BUNN, H. F., GALLOP, P. M.: Glycosylated hemoglobins and long-term blood glucose control in diabetes mellitus. M. Clin. Endocrinol. Metab. 44, 859-864 (1977).

14. DOLHOFER, R., STÄDELE, A. WIELAND O. H.: Clinical and biochemical studies on the . significance and formation of hemoglobins $A_{1 c}$ and $A_{10+b}$ in diabetes mellitus. Klinische
Wochenschrift 55, 945-954 (1977).

15. SVENDSEN, . A., CHRISTIANSEN, J. $S$.

lation of hemoglobin. Lancet 17, $\mathrm{N}$.: In vitro und in vivo Untersuchungen über Hämoglobin 16. N1. Kongress der Deutschen Diabetes-Gesellschaft in Freiburg i. Br. (1979).

$A_{1 c}$. 14. Kongress der Deutschen Diabetes-Gesellschaft in Freiburg i. Br. (1979). 17. SACHSE, G., KOCH, U., LAUBE, H., FEDERLIN, K.: HbA $1,0-c$ - Wirklich ein aussagekräftiger Langzeitparameter zur Beurteilung der diabet. Stoffwechsellage? - Einfluß von

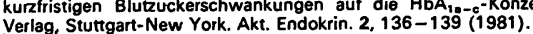

Verlag, Stuttgart-New York. Akt. Endokrin. 2, 136-139 (1981). Soforthilfe für statistische Tests mit wenigen Meßdaten. B. J.-Wissenschaftsverlag. Mannheim-Wien-Zürich (1972).

19. BUNN, H. F., HANEY, D. N., KAMIN, S., GABBAY, K. H., GALLOP, P. M.: The biosynthesis of human hemoglobin $A_{1 c}$ (slow glycosylation of hemoglobin in vivo). J. Clin. Invest. 57, 1652-1659 (1976a).

20. BUNN. H. F. HANEY, D. N.: Glycosylation of hemoglobin in vitro: affinity labeling of hemoglobin by glucose-6-phosphat. Proc. Natl. Acad. Sci. U.S.A. 73, 3534-3538 (1976b).

Anschrift der Verfasser

Dr. G. Sachse

Dr. A. Schornstein

Dr. H. Laube

Prof. Dr. K. Federlin

III. Medizinische Universitätsklinik und Poliklinik

Rodthohl 6

D.6300 Gießen 